\title{
ABM11 PDFs and the cross section benchmarks in NNLO
}

\section{Sergey Alekhin*}

DESY, Platanenallee 6, D-15738 Zeuthen, Germany

Institute for High Energy Physics, 142281 Protvino, Moscow region, Russia

E-mail: sergey.alekhin@desy.de

\section{Johannes Blümlein}

DESY, Platanenallee 6, D-15738 Zeuthen, Germany

E-mail: johannes.bluemlein@desy.de

\section{Sven-Olaf Moch}

II. Institut für Theoretische Physik, Universität Hamburg Luruper Chaussee 149, D-22761

Hamburg, Germany

DESY, Platanenallee 6, D-15738 Zeuthen, Germany

E-mail: sven-olaf.moch@desy.de

\begin{abstract}
We report an updated version of the ABKM09 NNLO PDF fit, which includes the most recent HERA collider data on the inclusive cross sections and an improved treatment of the heavy-quark contribution to deep-inelastic scattering using advantages of the running-mass definition for the heavy quarks. The ABM11 PDFs obtained from the updated fit are in a good agreement with the recent LHC data on the $W$ - and $Z$-production within the experimental and PDF uncertainties. We also perform a determination of the strong coupling constant $\alpha_{s}$ in a variant of the ABM11 fit insensitive to the influence of the higher twist terms and find the value of $\alpha_{s}=0.1133$ (11) which is in good agreement with the nominal ABM11 one and our earlier determination.
\end{abstract}

Loops and Legs in Quantum Field Theory - 11th DESY Workshop on Elementary Particle Physics, April 15-20, 2012

Wernigerode, Germany

\footnotetext{
* Speaker.
} 
Modern phenomenology of high-energy hadronic interactions in great extent is driven by the precise calculations, which include higher order perturbative QCD corrections and require accurate knowledge of the non-perturbative input coming from the parton distribution functions (PDFs). In turn the latter are steadily improved due to the progress in the calculations of the perturbative corrections, which are commonly used to tune the PDFs, and refined measurements of those processes. The ABKM09 PDFs [1] were also recently updated to the ABM11 ones [2] taking advantage of more accurate deep-inelastic-scattering (DIS) data obtained by the HERA experiments $[3,4]$ and improved theoretical treatment of the heavy-quark electro-production. This improvement is particularly important for the low- $x$ DIS data used in the analysis due to a significant contribution of the heavy quarks in the inclusive cross sections. In the ABKM09 fit it is computed within the 3-flavor factorization scheme with the heavy quarks appearing in the final state. The ABM11 fit is also based on the 3-flavor scheme, however, the $\overline{\mathrm{MS}}$-mass definition is now used for the charm and bottom quarks, in contrast to the pole-mass definition employed in the ABKM09 fit. This approach provides improved convergence of the perturbative QCD calculations for the heavy-quark electro-production [5] which is crucial in view of the full NNLO corrections to this process are still missing. The quality of the ABM11 fit for the newly appended HERA data is quite good. The value of $\chi^{2}=537$ is obtained for the combined H1\&ZEUS sample [3] including the neutral-current and the charged-current cross section data with the total number of data points (NDP) equal to 486, while for the neutralcurrent data collected by the $\mathrm{H} 1$ experiment in the high-inelasticity run [4] the value of $\chi^{2} / N D P=$ $137 / 130$. The latter data are particularly sensitive

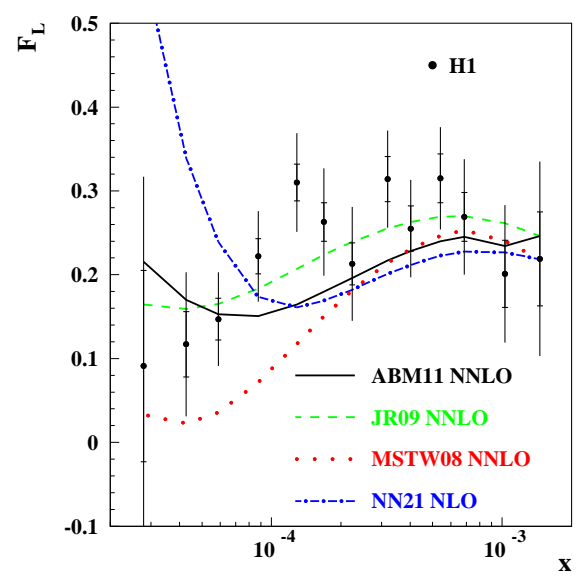

Figure 1: The data on $F_{L}$ versus $x$ obtained by the $\mathrm{H} 1$ collaboration [4] confronted with the 3flavor scheme NNLO predictions based on the different PDFs (solid line: this analysis, dashes: JR09 [6], dots: MSTW [7]). The NLO predictions based on the 3-flavor NN21 PDFs [8] are given for comparison (dashed dots). The value of $Q^{2}$ for the data points and the curves in the plot rises with $x$ in the range of $1.5 \div 45 \mathrm{GeV}^{2}$. to the longitudinal structure function $F_{L}$ at small $x$, which is in turn sensitive to the small- $x$ gluon distribution. The 3-flavor scheme NNLO-predictions based on the ABM11, JR09, MSTW08, and NN21 PDFs are compared with the $F_{L}$ measurements of Ref. [4] in Fig. 1. While the ABM11 and JR09 analyses are based on the 3-flavor treatment of the DIS, the MSTW08 and NN21 PDFs are obtained with different variants of the variable-flavor-number (VFN) scheme with the charm quarks appearing in the initial state. Therefore in order to provide a consistent comparison the MSTW08 and NN21 predictions are computed employing the complementary 3-flavor PDF sets provided by those groups. With such a unification the spread of the predictions in Fig. 1 displays the difference in the corresponding PDFs, basically the small- $x$ gluon distributions. While the PDFs obtained within the 3-flavor scheme provide a better description of the data, the use of a modeling based on the VFN scheme apparently leads to departures at low values of $Q^{2}$.

In any case the small- $x$ gluons by different groups can evidently consolidate using these data. 

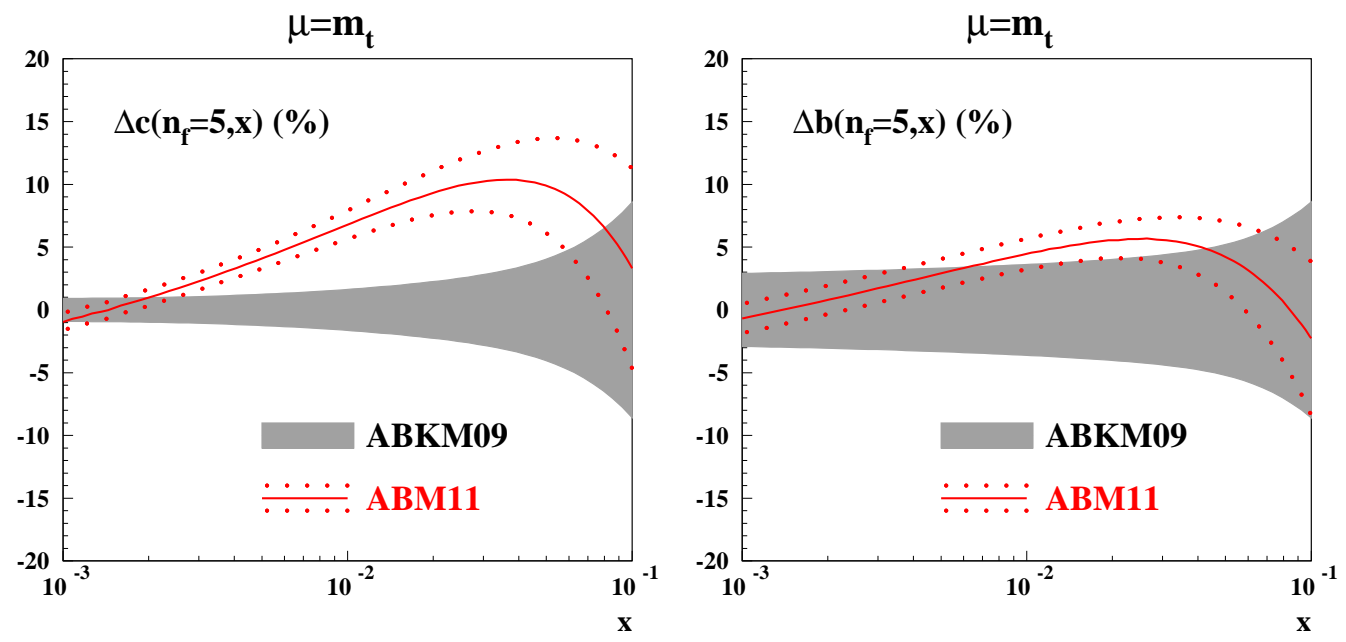

Figure 2: The charm- (left) and the bottom-quark (right) PDFs obtained in the fit: The dotted (red) lines denote the $\pm 1 \sigma$ band of relative uncertainties (in percent) and the solid (red) line indicates the central prediction resulting from the fit with the running masses taken at the PDG values. For comparison the shaded (grey) area represents the results of ABKM09 [1].

The DIS HERA data are sensitive to the value of the charm-quark mass $m_{c}$. Its $\overline{\mathrm{MS}}$-value is obtained by $m_{c}\left(m_{c}\right)=1.24 \pm 0.03$ (exp) ${ }_{-0.02}^{+0.03}$ (scale) ${ }_{-0.07}^{+0.00}$ (theory) $\mathrm{GeV}$ [9] preferred by the inclusive HERA data of Refs. [3,4] combined with the semi-inclusive data on open charm production [11] is in good agreement with the world average [10]. This provides an additional argument in favor of the 3-flavor description of DIS. Furthermore, in the ABM11 analysis the accuracy of the small- $x$ 4- and 5-flavor heavy-quark PDFs is improved as compared to the ABKM09 one due to the constraints on charm- and bottom-quark masses because the world average can be imposed, cf. Fig. 2. In contrast, the values of $m_{c}$ extracted from the HERA data within different variants of the VFN scheme demonstrate a wide spread and, moreover, they are systematically lower than the ones obtained from the $e^{+} e^{-}$data [11].

The value of the strong coupling constant $\alpha_{\mathrm{s}}\left(M_{\mathrm{Z}}\right)=0.1134(8)$ obtained in the NNLO ABM11 fit is in agreement with the earlier one of ABKM09. Meanwhile the NMC and SLAC DIS data sets prefer smaller and bigger value of $\alpha_{\mathrm{s}}$, respectively, cf. Fig. 3. Note that these two data sets are sensitive to the contribution of the higher twist terms [2,12], while the HERA and BCDMS data do not suffer from this contribution due to larger typical values of the momentum transfer $Q^{2}$ involved. To avoid the influence of higher twist terms we perform a variant of the ABM11 fit with the SLAC and NMC data dropped and the higher twist terms set to zero. The value of $\alpha_{\mathrm{s}}\left(M_{\mathrm{Z}}\right)=0.1133(11)$ obtained in this way is in a good agreement with the nominal value of ABM11 that demonstrates a consistent treatment of the higher twist terms in the present analysis. The NNLO ABM11 value of $\alpha_{\mathrm{s}}\left(M_{\mathrm{Z}}\right)$ is essentially lower than the PDG world average [10]. On the other hand the ABM11 value of $\alpha_{\mathrm{S}}\left(M_{\mathrm{Z}}\right)$ is in agreement with the recent NNLO determinations obtained from the analysis of thrust distributions measured in the $e^{+} e^{-}$collisions with account of the power corrections $[13,14]$ and also the lattice calculation of the QCD static energy [15].

Benchmarks for the rates of key-processes at Tevatron and the LHC were given in $[1,2,16]$. 


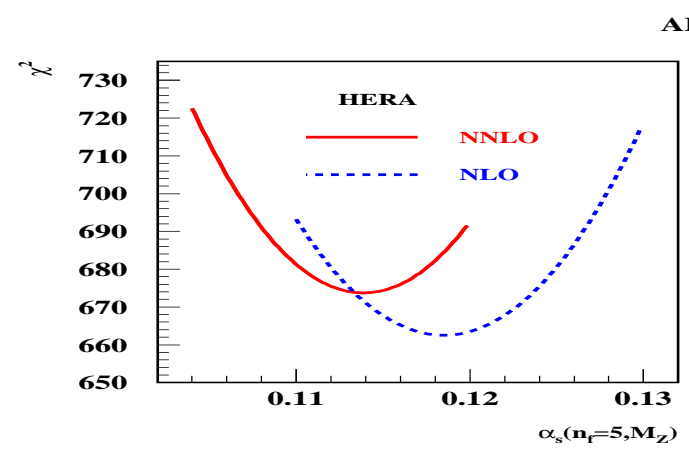

ABMI1
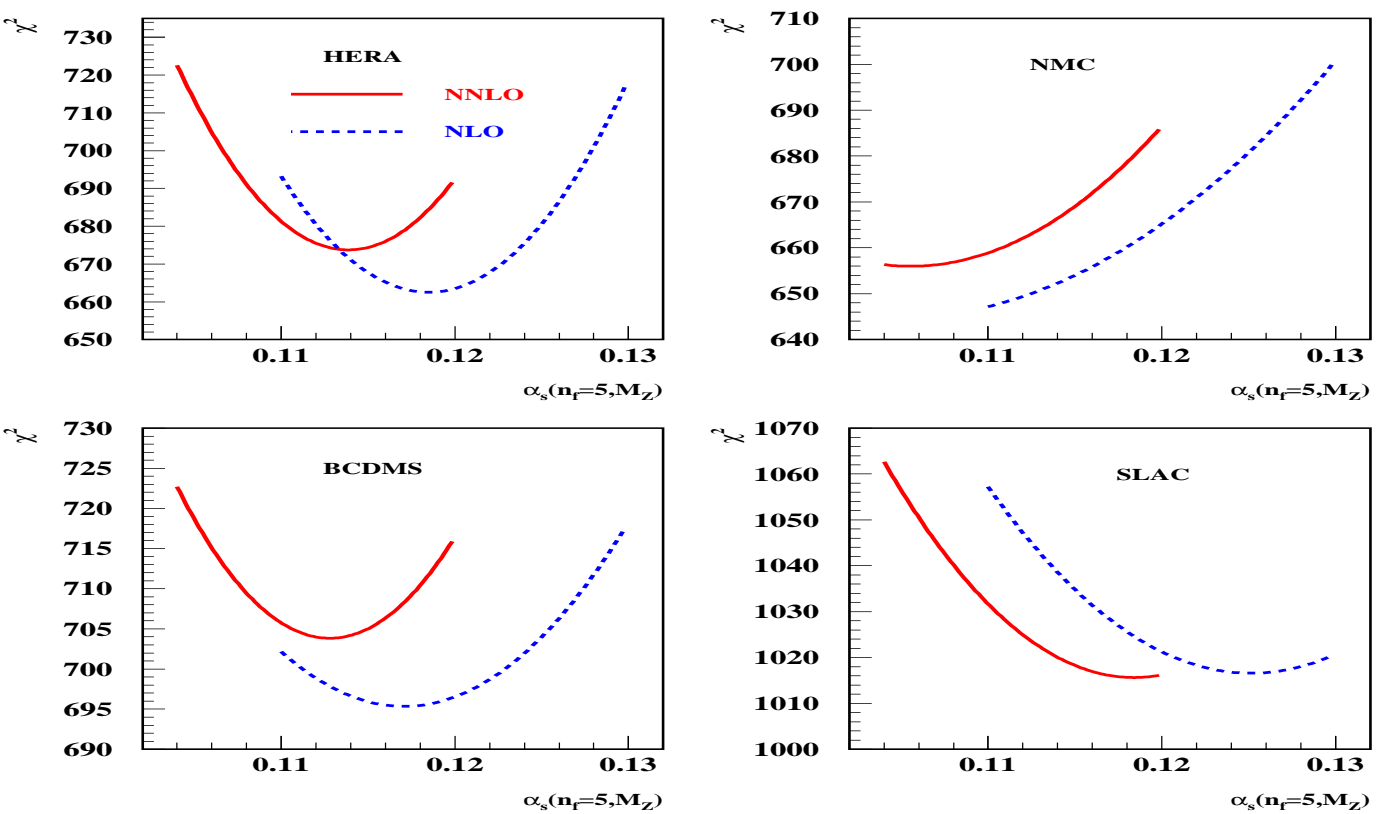

Figure 3: The $\chi^{2}$-profile versus the value of $\alpha_{s}\left(M_{Z}\right)$, for the separate data subsets, all obtained in variants of the present analysis with the value of $\alpha_{s}$ fixed and all other parameters fitted (solid lines: NNLO fit, dashes: NLO fit).

For the first LHC data on the Drell-Yan differential cross sections [17-20] we observe good agreement with the predictions based on the ABM11 PDFs. These data were obtained by detecting the leptonic decays of $W$ - and $Z$-bosons with the charged lepton pseudo-rapidities in the range of $-2.5 \div 2.5,-2.4 \div 2.4$, and $2 \div 4.5$ for the ATLAS, CMS, and LHCb experiments, respectively. The data on the Z-boson pseudo-rapidities and on the charged lepton pseudo-rapidities for the case of $W$-boson production are confronted to the NNLO predictions obtained with ABM11 PDFs in Figs. 4-7. The central values of the predictions are obtained using a fully exclusive code DYNNLO 1.3 [21]. However, the uncertainties due to PDFs are computed with another fully exclusive code FEWZ 3.1 [22], which allows to estimate the variation of the cross section with the PDFs without accumulating the Monte-Carlo integration errors in the cross section differences. The typical accuracy of the DYNNLO results displayed in Figs. 4-7 is better than 1\% and it does not exceed the data errors.

Agreement between the data and the predictions is quantified by the following $\chi^{2}$ functional

$$
\chi^{2}=\sum_{i, j}\left(y_{i}-f_{i}^{(0)}\right)\left[C^{-1}\right]_{i j}\left(y_{j}-f_{j}^{(0)}\right),
$$

with the covariance matrix

$$
C_{i j}=C_{i j}^{e x p}+\sum_{k=1}^{28}\left(f_{i}^{(k)}-f_{i}^{(0)}\right)\left(f_{j}^{(k)}-f_{j}^{(0)}\right) .
$$

The first term in Eq. (2) corresponds to the covariance matrix describing the errors in the experimental data, while the second term appears due to the PDF uncertainties. The latter contribute 
$\operatorname{ATLAS}(7 \mathrm{TeV}, 35$ 1/pb)
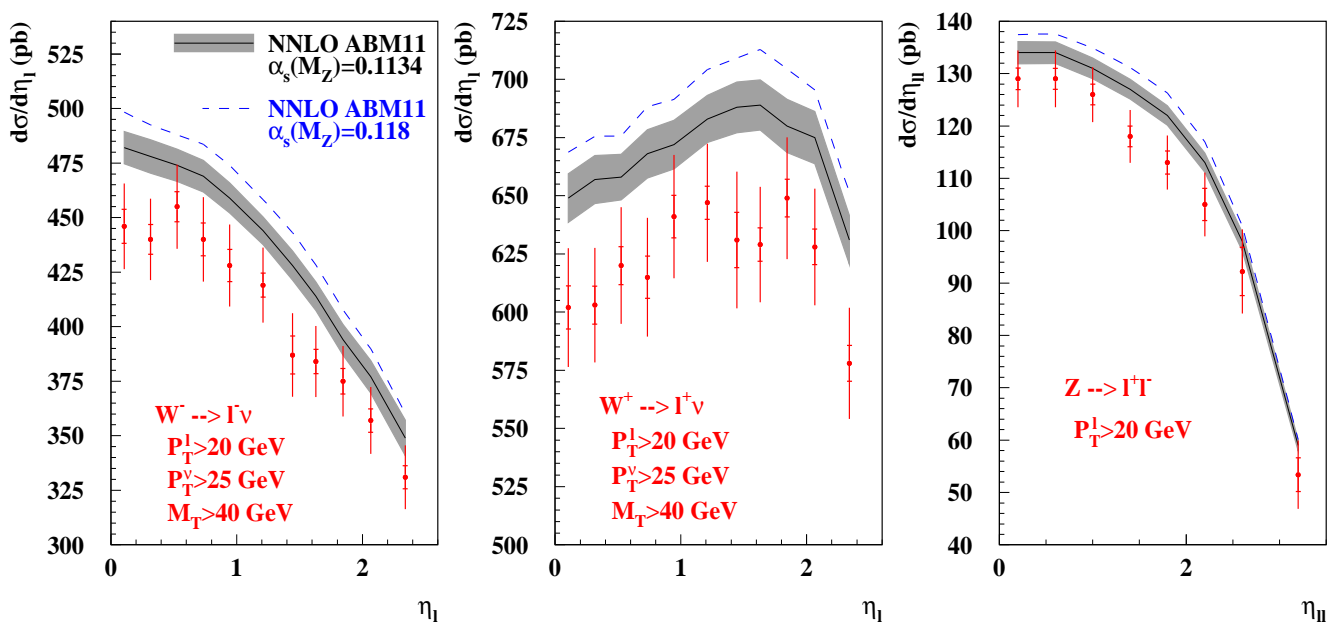

Figure 4: The ATLAS data of Ref. [17] on the rapidity distribution of the charged leptons $l^{-}$(left panel), $l^{+}$(central panel), and Z-boson (right panel) in comparison with the NNLO predictions computed with the ABM11 PDFs taking into account the uncertainties due to PDFs (shaded area). The dashed curves display the ABM11 predictions obtained with the value of $\alpha_{s}\left(M_{Z}\right)=0.118$.

to the covariance matrix by virtue of the differences between predictions obtained for the central ABM11 PDF set, $f_{j}^{(0)}$, and the ones for each of the 28 ABM11 PDF sets representing the PDF uncertainties, $f_{j}^{(k)}$. The experimental covariance matrix for the ATLAS data of Ref. [17] is computed by

$$
C_{i j}^{e x p}=\delta_{i j} \sigma_{i}^{2}+f_{i}^{(0)} f_{j}^{(0)} \sum_{k=1}^{31} s_{i}^{k} s_{j}^{k}
$$

where $\sigma_{i}$ are the statistical errors in the data combined in quadrature with the uncorrelated errors, $s_{i}^{k}$ are the correlated systematic uncertainties representing 31 independent sources including the normalization, and $\delta_{i j}$ is the Kronecker symbol. In view of the small background for the $W$ and $Z$-production signal all systematic errors are considered as multiplicative. Therefore, they are weighted with the theoretical predictions $f_{i}^{(0)}$. The experimental covariance matrices for the CMS and LHCb data of Refs. [18-20] are available in publications and they are directly employed in Eq. (2) after re-weighting by the theoretical predictions similarly to Eq. (3) with the normalization uncertainty taken into account in the same way as for the ATLAS data.

The values of $\chi^{2}$ for different LHC data samples are summarized in Table 1. Due to an inconsistency in the covariance matrix for the LHCb Z-boson data [23] quoted in Ref. [19] the Z-boson data are not employed in the calculation of $\chi^{2}$ for this LHCb sample. In general the values of $\chi^{2} / N D P$ in Table 1 are comparable with 1 within possible statistical fluctuations. The ATLAS data [17] systematically undershoot the predictions, although the statistical discrepancy of the disagreement is not significant in view of still relatively large experimental uncertainties. Moreover, it is worth noting that the recent high-luminosity LHCb data [20] on the Z-boson production are in much better agreement with the ABM11 predictions. In particular this is also observed for the rapidity range overlapping with the corresponding ATLAS data that may indicate a potential in- 


\section{CMS (7 TeV, 840 1/pb)}

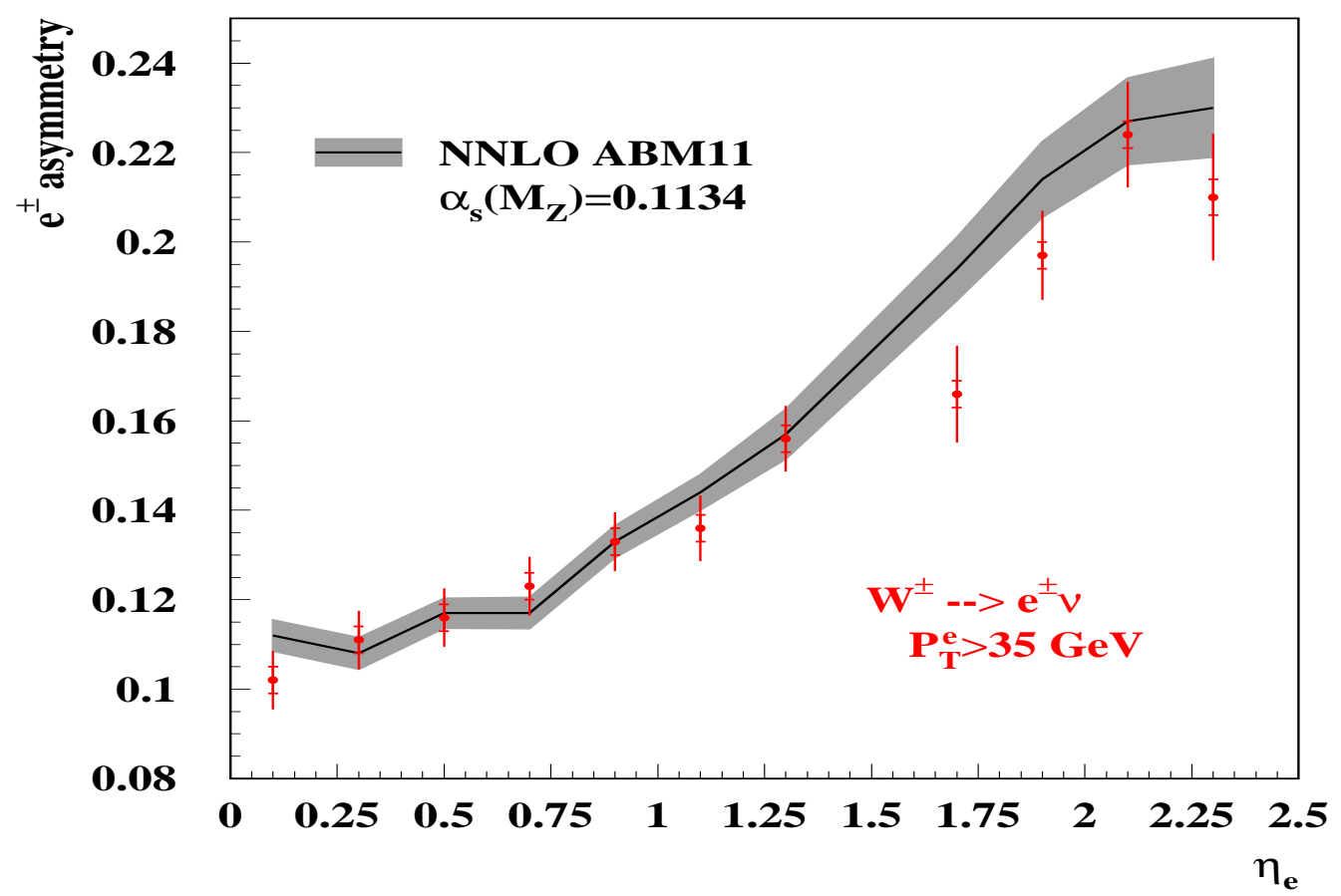

Figure 5: The same as Fig. 4 for the CMS data of Ref. [18] on the electron asymmetry rapidity distribution. The curve corresponding to the ABM11 predictions obtained with the value of $\alpha_{S}\left(M_{Z}\right)=0.118$ coincides with the nominal one.

consistency between the ATLAS and LHCb normalization. The ABM11 predictions demonstrate a certain tension with the earlier $\mathrm{LHCb}$ data [19] on the $W^{+}$-boson production at moderate rapidity, however, the overall agreement is satisfactory. If, nonetheless, the discrepancies with the ATLAS and LHCb data being observed are confirmed with better statistical significance, the ABM11 quark distributions at $x \sim 0.01$ would have to be lowered in order to accommodate those data in a fit. Meanwhile, the charged-lepton asymmetry which is less sensitive to the experimental uncertainties is in a good agreement with the ABM11 predictions both for the ATLAS and CMS data.

\begin{tabular}{|c|c|c|c|c|}
\hline Experiment & ATLAS [17] & CMS [18] & LHCb [19] & LHCb [20] \\
\hline Final states & $W^{+} \rightarrow l^{+} v$ & $W^{+} \rightarrow e^{+} v$ & $W^{+} \rightarrow \mu^{+} v$ & $Z \rightarrow e^{+} e^{-}$ \\
& $\begin{array}{c}W^{-} \rightarrow l^{-} v \\
Z \rightarrow l^{+} l^{-}\end{array}$ & $W^{-} \rightarrow e^{-} v$ & $W^{-} \rightarrow \mu^{-} v$ & \\
\hline Luminosity (1/pb) & 35 & 840 & 37 & 940 \\
\hline$N D P$ & 30 & 11 & 10 & 9 \\
\hline$\chi^{2}$ & $34.5(7.7)$ & $11.8(4.7)$ & $13.0(4.5)$ & $11.5(4.2)$ \\
\hline
\end{tabular}

Table 1: The value of $\chi^{2}$ obtained for different samples of the Drell-Yan LHC data with the NNLO ABM11 PDFs. The figures in parenthesis give one standard deviation of $\chi^{2}$ equal to $\sqrt{2 N D P}$. 
LHCb (7 TeV, 37 1/pb)
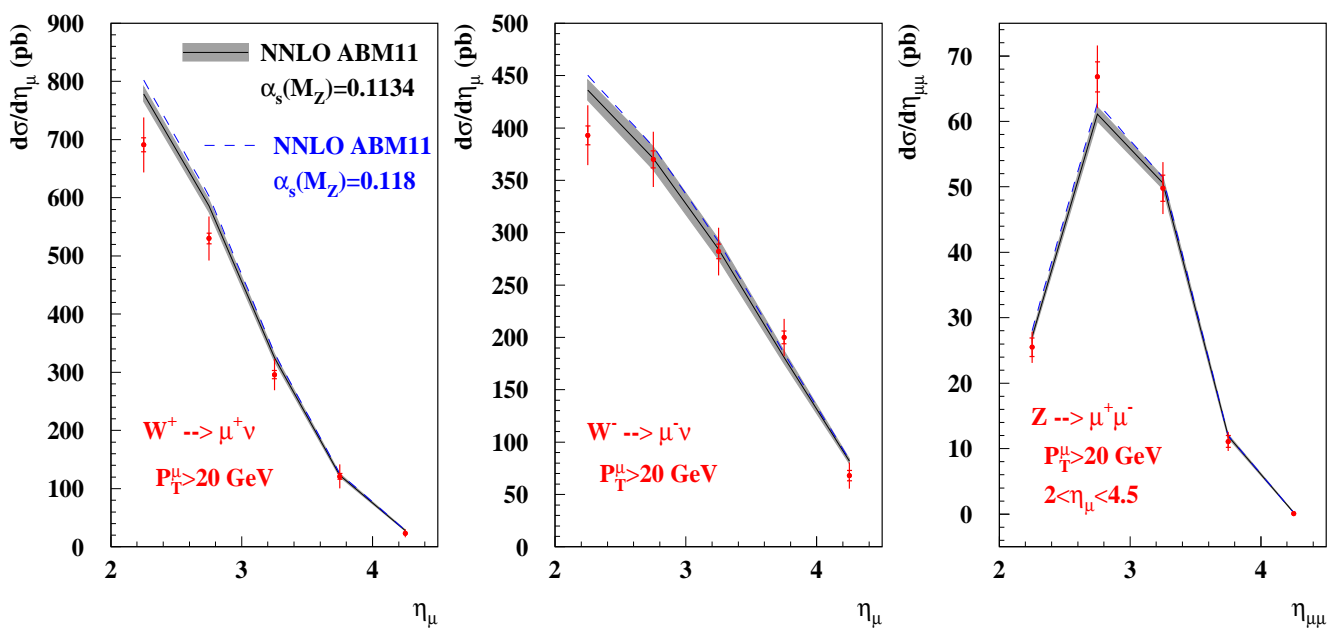

Figure 6: The same as Fig. 4 for the LHCb data of Ref. [19] on the rapidity distribution of $\mu^{+}$(left panel), $\mu^{-}$(central panel), and Z-boson (right panel).

The LHC Drell-Yan data were confronted to the predictions based on the different PDFs including ABM11 ones in Ref. [24] earlier. In contrast with our analysis the NNLO predictions of Ref. [24] are obtained using the NLO MCFM code [25] supplemented by the NNLO K-factors which were computed separately with the DYNNLO code. Furthermore, the benchmarking of Ref. [24] is performed using the PDFs corresponding to the value of $\alpha_{s}\left(M_{Z}\right)=0.118$. This value is disfavored by the ABM11 fit. Nonetheless, in order to allow a consistent comparison with the benchmarking of Ref. [24], we also provide the ABM11 prediction corresponding to the value of $\alpha_{s}\left(M_{Z}\right)=0.118$. The predictions obtained in this way are compared with the data and the nominal ABM11 predictions in Figs. 4,5,6,7. The charged-lepton asymmetry does not depend on the $\alpha_{s}$ value, while the shift in the $W$ and $Z$ absolute cross-sections obtained with this variation of $\alpha_{s}$ is of the order of the uncertainties due to PDFs. The value of $\chi^{2}=14.3$ obtained for the LHCb data [19] with the enhanced value of $\alpha_{s}$ is within the statistically allowed range. Meanwhile the value of $\chi^{2}$ obtained in this case for the ATLAS data [17] is 40.0, i.e. slightly above the $1 \sigma$ statistical margin. This is obviously related to the offset of the nominal ABM11 predictions w.r.t. the ATLAS data. Therefore the final conclusion about the significance of this excess can be drawn only after the normalization discrepancy for ATLAS and LHCb is clarified. Anyway, both values are significantly smaller than those quoted for the ABM11 benchmarking in Ref. [24]. In particular this happens due to the PDF uncertainties were not taken into account in the statistical analysis of Ref. [24]. The central values for the ABM11 predictions obtained in that benchmarking also do not agree with ours in places, cf. Fig. 12 in Ref. [24] and Fig. 4 in the present paper ${ }^{1}$. The recent LHCb data on the $Z \rightarrow e^{+} e^{-}$production were not included into the benchmarking of Ref. [24], however, they are compared with the NNLO predictions based on the CT10 [26], MSTW09 [7], and NN21 [27] PDFs in Fig. 4 of Ref. [20]. These PDFs provide consistent results. Nonetheless all three undershoot the

\footnotetext{
${ }^{1}$ To facilitate further clarification of the discrepancy the tabulated NNLO ABM11 predictions for the available ATLAS, CMS, and LHCb Drell-Yan data are attached to the arXiv version of these Proceedings.
} 


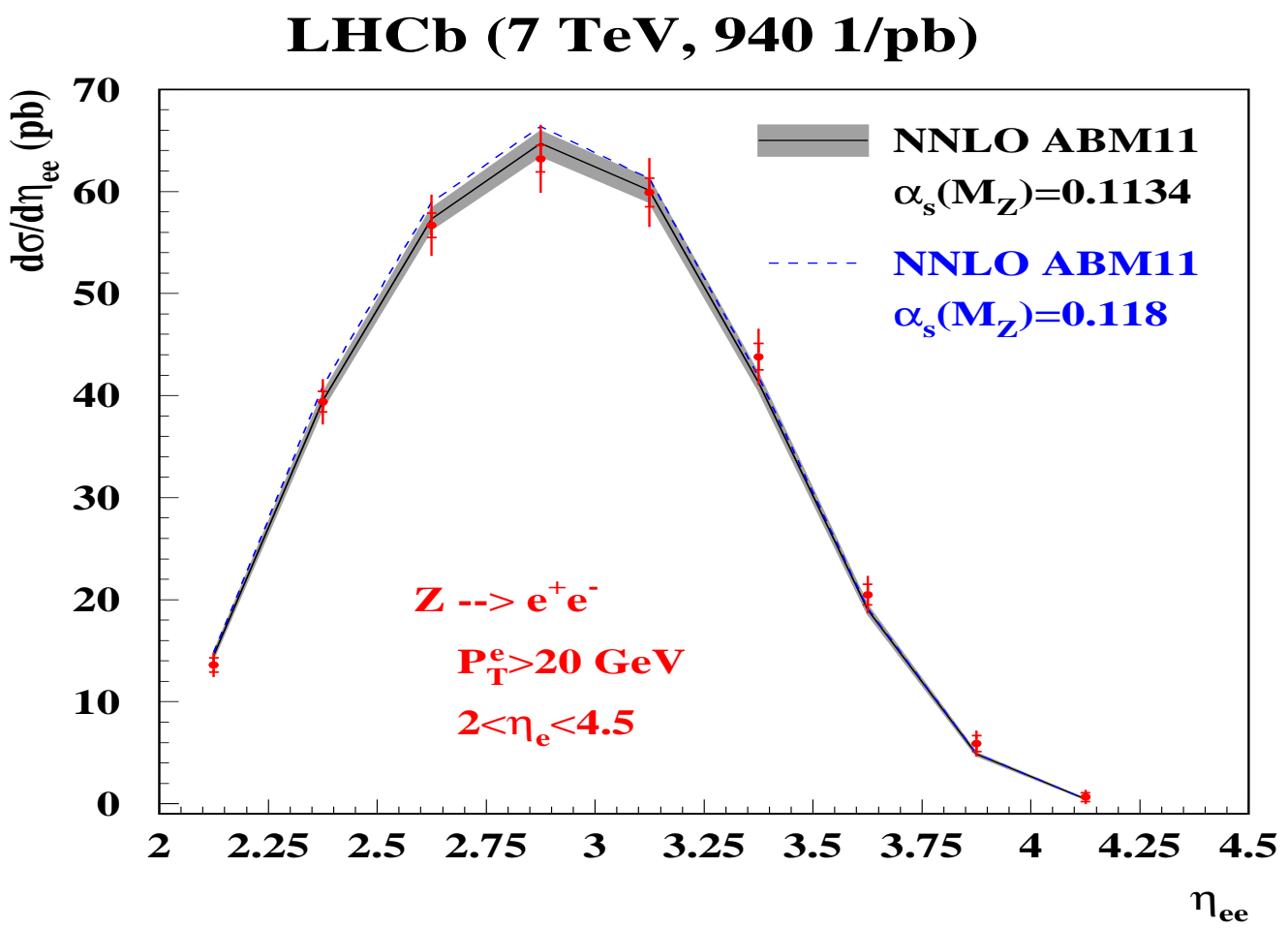

Figure 7: The same as Fig. 4 for the LHCb data of Ref. [20] on the Z-boson rapidity distribution.

LHCb Z-boson data at large rapidity. To quantify this disagreement we check $\chi^{2}$ for these data taking NNLO MSTW08 predictions with account of the PDF uncertainties and find the value of 27.9, more than twice bigger than ours, cf. Table 1.

In summary, we present the updated version of the NNLO PDF fit based on the combination of the DIS and Drell-Yan data. The update includes the most recent HERA collider data on the inclusive neutral-current and charged-current cross sections. From the theory side we improved the treatment of the heavy-quark contribution to DIS using advantages of the running-mass definition. The ABM11 PDFs obtained from the updated fit are in a good agreement with the recent LHC data on the $W$ - and $Z$-production within the experimental and PDF uncertainties. Our results do not confirm the benchmarking of the ABM11 PDFs published elsewhere [24]. We also performed a determination of the $\alpha_{s}$ value in a variant of the ABM11 fit insensitive to the higher twist terms and find a value, which is in very good agreement with the nominal one and our earlier determination of $\alpha_{s}$.

Acknowledgments. We acknowledge fruitful discussions concerning the Drell-Yan LHC data with Uta Klein and Katharina Müller. We are also grateful to Juan Rojo for clarification of some details of Ref. [24].

\section{References}

[1] S. Alekhin, J. Blümlein, S. Klein and S. Moch, Phys. Rev. D 81 (2010) 014032 [arXiv:0908.2766 [hep-ph]]. 
[2] S. Alekhin, J. Blümlein and S. Moch, Phys. Rev. D 86 (2012) 054009 [arXiv:1202.2281 [hep-ph]].

[3] F. D. Aaron et al. [H1 and ZEUS Collaboration], JHEP 1001 (2010) 109 [arXiv:0911.0884 [hep-ex]].

[4] F. D. Aaron et al. [ H1 Collaboration], Eur. Phys. J. C 71 (2011) 1579 [arXiv:1012.4355 [hep-ex]].

[5] S. Alekhin and S. Moch, Phys. Lett. B 699 (2011) 345 [arXiv:1011.5790 [hep-ph]].

[6] P. Jimenez-Delgado and E. Reya, Phys. Rev. D 79 (2009) 074023 [arXiv:0810.4274 [hep-ph]].

[7] A. D. Martin, W. J. Stirling, R. S. Thorne and G. Watt, Eur. Phys. J. C 63 (2009) 189 [arXiv:0901.0002 [hep-ph]].

[8] R. D. Ball, V. Bertone, F. Cerutti, L. Del Debbio, S. Forte, A. Guffanti, J. I. Latorre and J. Rojo et al., Nucl. Phys. B 849 (2011) 296 [arXiv:1101.1300 [hep-ph]].

[9] S. Alekhin, J. Blümlein, K. Daum, K. Lipka and S. Moch, arXiv:1212.2355 [hep-ph].

[10] J. Beringer et al. [Particle Data Group Collaboration], Phys. Rev. D 86 (2012) 010001.

[11] H. Abramowicz et al. [H1 and ZEUS Collaborations], arXiv:1211.1182 [hep-ex].

[12] S. Alekhin, J. Blümlein and S. Moch, Eur. Phys. J. C 71 (2011) 1723 [arXiv:1101.5261 [hep-ph]].

[13] R. Abbate, M. Fickinger, A. H. Hoang, V. Mateu and I. W. Stewart, Phys. Rev. D 86 (2012) 094002 [arXiv:1204.5746 [hep-ph]].

[14] T. Gehrmann, G. Luisoni and P. F. Monni, arXiv:1210.6945 [hep-ph].

[15] A. Bazavov, N. Brambilla, X. Garcia i Tormo, P. Petreczky, J. Soto and A. Vairo, Phys. Rev. D 86 (2012) 114031 [arXiv:1205.6155 [hep-ph]].

[16] S. Alekhin, J. Blümlein, P. Jimenez-Delgado, S. Moch and E. Reya, Phys. Lett. B 697 (2011) 127 [arXiv:1011.6259 [hep-ph]].

[17] G. Aad et al. [ATLAS Collaboration], Phys. Rev. D 85, 072004 (2012) [arXiv:1109.5141 [hep-ex]].

[18] S. Chatrchyan et al. [CMS Collaboration], Phys. Rev. Lett. 109 (2012) 111806 [arXiv:1206.2598 [hep-ex]].

[19] R. Aaij et al. [LHCb Collaboration], JHEP 1206 (2012) 058 [arXiv:1204.1620 [hep-ex]].

[20] R. Aaij et al. [ LHCb Collaboration], arXiv:1212.4620 [hep-ex].

[21] S. Catani, L. Cieri, G. Ferrera, D. de Florian and M. Grazzini, Phys. Rev. Lett. 103 (2009) 082001 [arXiv:0903.2120 [hep-ph]]; S. Catani and M. Grazzini, Phys. Rev. Lett. 98 (2007) 222002 [hep-ph/0703012].

[22] Y. Li and F. Petriello, Phys. Rev. D 86 (2012) 094034 [arXiv:1208.5967 [hep-ph]].

[23] K.Müller, private communication.

[24] R. D. Ball, S. Carrazza, L. Del Debbio, S. Forte, J. Gao, N. Hartland, J. Huston and P. Nadolsky et al., arXiv:1211.5142 [hep-ph].

[25] http://mcfm.fnal.gov/

[26] P. Nadolsky, J. Gao, M. Guzzi, J. Huston, H. -L. Lai, Z. Li, J. Pumplin and D. Stump et al., arXiv:1206.3321 [hep-ph].

[27] R. D. Ball et al. [NNPDF Collaboration], Nucl. Phys. B 855 (2012) 153 [arXiv:1107.2652 [hep-ph]]. 